\title{
Análisis del Comportamiento de Robots Móviles con RNA. Un Acercamiento desde el Paradigma Reactivo
}

\author{
Alejandro Hossian, Gustavo Monte, Verónica Olivera \\ Facultad Regional del Neuquén \\ Universidad Tecnológica Nacional \\ Plaza Huincul, Neuquén, Argentina \\ alejandrohossian@yahoo.com.ar, verolivera@gmail.com
}

\begin{abstract}
Resumen. Por medio de la navegación es posible guiar el curso de un robot móvil a través de un entorno con presencia de obstáculos. Se conocen diferentes esquemas para llevar a cabo esta tarea, pero todos ellos tienen el objetivo común de dirigir el vehículo hacia su destino de la manera más segura y eficiente posible. La capacidad de reacción que pueda poseer el robot cuando se encuentra ante situaciones inesperadas, debe constituir su cualidad más distintiva para desenvolverse eficazmente en el entorno donde este deba operar, lo cual indica el grado de autonomía que este posee. La navegación robótica es aplicable a múltiples disciplinas y entornos industriales; $y$ en este sentido, la aplicación de la Inteligencia Artificial a través de las diferentes tecnologías inteligentes que la componen (redes neuronales, algoritmos genéticos y aprendizaje automático entre otras) cobra un gran protagonismo dentro del campo de la Robótica Cognitiva para su desarrollo. El objetivo principal del presente trabajo se focaliza en el análisis $y$ presentación de resultados de investigación en técnicas de navegación robótica, que se han obtenidos en base a experimentos llevados a cabo mediante la aplicación de la tecnología de las redes neuronales artificiales, las cuales concentran las mejores características del paradigma reactivo en lo que se refiere a la navegación autónoma de robots. En el presente artículo se evalúa la efectividad y el desempeño de este paradigma mediante la aplicación de una red neuronal de arquitectura sencilla, obteniendo conclusiones en lo que respecta a la conducta deseada del robot y comparándola con la que manifiesta en su desempeño. Los parámetros que se analizan para esta evaluación están relacionados con la evitación de obstáculos, velocidad de respuesta, optimización de las trayectorias que adopta y el logro de los objetivos.
\end{abstract}

\section{Palabras Clave: Navegación Robótica, Redes Neuronales} Artificiales, Paradigma Reactivo, Robótica Autónoma.

\section{INTRODUCCIÓN}

La robótica autónoma constituye un área muy particular en el vasto campo de la robótica. En esta línea, puede afirmarse que para que un robot sea autónomo es necesario que el mismo sea capaz de reaccionar ante situaciones que no han sido consideradas en la programación de su control y sin ningún tipo de supervisión proveniente del exterior.

Aún así, y considerando que su control está definido por un programa determinado, el vehículo debe tener la capacidad suficiente para realizar las tareas que le fueron encomendadas sin que sea necesario que su programa de control defina en forma explícita todas las acciones posibles que este debería realizar ante la totalidad de situaciones posibles que pueden tener lugar en su entorno [1].

Lo hasta aquí expuesto, pone de manifiesto que el robot autónomo debe ser "no totalmente preprogramado". A partir de este enfoque, si se considera a un robot operando en un proceso de ensamblado o pintura y se le cambia alguna condición específica de operación, tal como puede ser el desplazamiento de la pieza a ensamblar, el robot ya no estará en condiciones de llevar a cabo estas tareas conforme a las condiciones de diseño. Es decir, dichos robots no tienen la capacidad de desarrollar su actividad ante situaciones que no fueron tenidas en cuenta en su programa de control.

En un principio, las investigaciones de robótica llevadas a cabo consideraba que los entornos en donde operaban los robots eran de tipo estructurado, entendiéndose por tal a aquellos que se los puede definir en forma precisa en lo que se refiere a su configuración (qué objetos existen, forma de los mismos, posición en la que se encuentran, etc), ya sea porque el ambiente permanece inalterable en el tiempo, o bien porque los cambios que puedan darse en el mismo son predecibles y, en consecuencia, pueden ser formalizados en términos computacionales. Entonces, se puede afirmar que el enfoque tradicional en robótica asume un perfecto conocimiento del entorno de operación del robot y de las consecuencias que conlleva cada acción que éste realiza, de modo que le permita al diseñador fijar políticas preactivas y de contingencia a los efectos de poder prever resultados no deseados.

Este acercamiento al problema resultó ser de suma utilidad en aquellos ambientes donde las llamadas "células de trabajo" se mantenían fijas en sus posiciones mientras el robot desarrollaba sus tareas. En la medida en que estos ambientes comenzaron a manifestar la necesidad de efectuar cambios para optimizar su producción, es que dicha aproximación pasa a caracterizarse por su rigidez y falta de flexibilidad, no siendo aplicable al caso de robots que deban operar en ambientes dinámicos, es decir, que son cambiantes en el tiempo y no totalmente conocidos, puesto que es muy difícil de prever los numerosos cambios que demandan estos entornos, o en caso de que se puedan prever, el volumen de información a procesar en este sentido sería demasiado grande. Por tal motivo, si se pretende disponer de un sistema de robot que sea capaz de operar en entornos no estructurados, el mismo no 
puede estar totalmente preprogramado y debe poseer una arquitectura cognitiva que le permita establecer las correspondientes relaciones entre lo que el robot percibe del entorno, sean las entradas a los sensores, y sus acciones sobre el mismo, las salidas de los actuadores o motores. Este hecho hace que el robot pueda generar de manera autónoma su mapa sensor - actuador y que tenga la capacidad de adaptarse a los cambios que ocurren en el ambiente de operación.

El presente trabajo centra su atención en la generación de este mapa en función de las características del ambiente de operación del robot, y que la información que éste brinda a través de dicho mapa se procese aplicando la tecnología de las Redes Neuronales Artificiales (RNA).

\section{MARCO TEÓRICO}

Si se busca un sistema robot con la capacidad de desarrollar su actividad en un entorno de características dinámicas y no conocidas en forma absoluta quien lo diseña, este debe contar con algún tipo de arquitectura cognitiva para su control que le permita establecer vinculaciones entre su sistema sensorial y las acciones que toma sobre el mundo en el cual está operando; esto le permitirá generar en forma autónoma el llamado "mapa sensor - actuador". En este sentido, es también importante que el sistema robot aprenda de sus errores y optimice su desempeño, motivo por el cual también estará capacitado para adaptarse a variaciones que tienen lugar en su ambiente operativo.

Durante los últimos años, las investigaciones de robótica muestran que dos son los enfoques que han servido de guía: el enfoque "basado en conocimiento", que tiene su origen en la inteligencia artificial simbólica, y más recientemente, el enfoque "basado en comportamientos", que se inspira en la naturaleza y toma distancia de algunas de las líneas tradicionales. A continuación se presentan algunas consideraciones acerca de los enfoques mencionados.

\section{A. Enfoque basado en conocimiento}

A fin de alcanzar la autonomía en los robots, se ha utilizado la inteligencia artificial simbólica y la piedra basal en la cual se sustenta, se focaliza en el hecho de que la inteligencia es intrínsicamente un fenómeno computacional, y en consecuencia, es posible estudiarla y aplicarla en diferentes sistemas, so pretexto de que los mismos se encuentren fuera de su entorno de actuación.

Este enfoque, también recibe el nombre de "deliberativo", está fundamentado en una visión introspectiva acerca de cómo piensan y actúan los seres humanos; dicho de otra forma, en relación con los "estados de ánimo" o de "conciencia". Un ejemplo de esto sería: "una persona que ve una puerta y decide pasar a través de ella".

Sintetizando, las características más salientes que presentan las arquitecturas de control enmarcadas dentro de este enfoque son las siguientes:

- Hacen uso de técnicas de razonamiento para decidir las acciones que el robot ha de seguir en base a un modelo del entorno; el procesamiento de la información se lleva a cabo en forma secuencial, de modo tal que el robot opera sobre el ambiente de navegación de acuerdo a las modificaciones que se producen en el modelo interno que este posee del ambiente.
- Conforme lo señalado anteriormente, las técnicas de razonamiento, implican estructuras de razonamiento en alto nivel que le permiten al robot hacer frente a requerimientos de navegación de alta complejidad.

- Estas arquitecturas parten de una descomposición de los procesos que el robot debe llevar a cabo en tareas independientes que luego se unifican; estas tareas son las siguientes: interpretación de la información relevada de los sensores, modelado del ambiente en el que el sistema de robot debe desempeñarse, modelo sobre el cuál se planifica un conjunto de acciones a desarrollar por el robot y, por último, estas acciones se realizan mediante un módulo de ejecución que establece los valores correspondientes a cada movimiento.

La figura 1 ilustra las arquitecturas de control del enfoque basado en conocimiento y sus correspondientes tareas.

Concluyendo, este enfoque admite que el robot percibe el mundo, planifica la acción que va a llevar a cabo y la ejecuta; es decir, la información percibida por el robot se representa en un modelo global del ambiente.

Pero también, uno de los principales problemas de este enfoque consiste en que algunos de estos procesos, como la sensorización y la planificación se realizan mediante módulos desarrollados sin considerar los otros módulos presentes en la arquitectura. Esta falta de interacción entre los diferentes módulos que conforman estas arquitecturas, constituyen la razón fundamental de la falta de robustez del enfoque basado en conocimiento. En consecuencia, cuando se acomete el diseño de una arquitectura cognitiva es interesante considerar tres dificultades que se pueden presentar [2]:

- Ocurre que, no es sencillo descomponer el sistema de control en todos los casos.

- Y en muchos casos las interacciones entre los módulos las determina el propio ambiente, y por ende, resultan ser más complejas que unos simples enlaces entre estos módulos.

Las interacciones entre los módulos aumenta de manera exponencial, a medida que crece la complejidad del sistema.

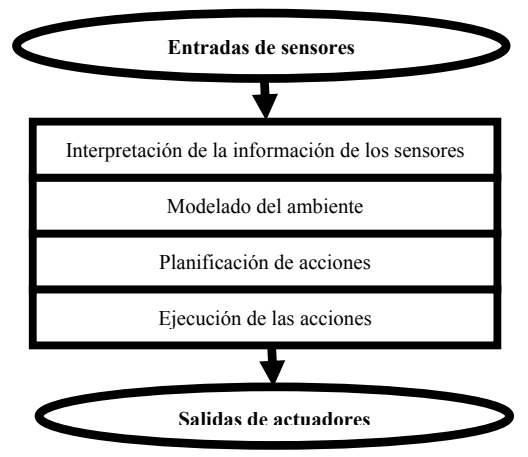

Fig. 1. Arquitectura de control que sigue el enfoque basado en conocimiento

\section{B. Enfoque reactivo}

En contraposición al enfoque anterior, el enfoque reactivo se basa en modelos biológicos para explicar el comportamiento observado en distintos organismos vivos. Las arquitecturas que siguen este tipo de comportamiento, a veces llamadas "puramente reactivas", implementan estrategias de control como una colección de pares "condiciones - acciones" 
y no llevan a cabo procesos de planificación ya que no operan sobre un modelo interno del ambiente.

La respuesta a estímulos es reflexiva, no regulada por procesos de carácter deliberativos de ningún tipo y los movimientos del robot se guían sólo a partir de la información que se está presente en ese momento en los sensores. Por ende, las acciones del robot se basan en un acoplamiento directo entre sensores y actuadores mediante bucles rápidos de realimentación.

A continuación, se repasan las características más sobresalientes de estas arquitecturas de control enmarcadas dentro de este enfoque:

- La velocidad en el procesamiento de información es mayor que las correspondientes al enfoque basado en conocimiento.

- Aún así, la gran velocidad de respuesta que poseen estas arquitecturas, presentan importantes limitaciones cuando el robot debe abordar tareas que requieren planificación.

- Los modelos de procesamiento de información "masivamente paralelos" son la base de estas arquitecturas, como por ejemplo, las redes neuronales artificiales (RNA).

Para cerrar este enfoque diremos que el robot percibe el mundo y ejecuta la acción que le parezca más adecuada; o sea, la información percibida por el robot no se integra en ningún modelo del ambiente. Es decir, el ambiente de operación es su mejor ambiente.

La figura 2 muestra las arquitecturas de control que siguen el enfoque reactivo puro.

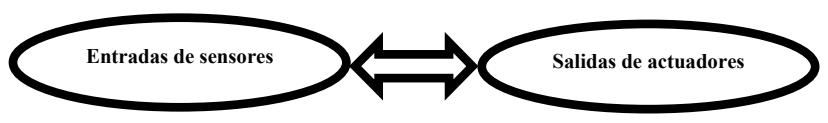

Fig. 2. Arquitectura de control que sigue el enfoque reactivo

\section{Enfoque basado en comportamientos ${ }^{l}$}

Una evolución de las reactivas puras son las arquitecturas que siguen este enfoque, en el sentido de que los comportamientos describen, de algún modo, la forma en que reacciona el sistema robot ante un determinado estado de los sensores; mientras que los actuadores determinan las acciones a seguir por parte del robot de una manera un poco más elaborada que la consulta a una simple tabla de decisión "sensor - actuador". Asimismo, estas arquitecturas funcionan en base a un conjunto de comportamientos paralelos ejecutándose en forma concurrente y donde cada uno de los distintos módulos de comportamiento se vinculan en forma directa con los sensores y actúan directamente sobre los actuadores [3]. Algunos ejemplos de comportamientos serían: buscar alimentos, cerrar pinzas o evitar obstáculos.

En relación a las dificultades que presentan los dos enfoques anteriores, el diseño aislado de los módulos individuales en el enfoque basado en conocimiento y la falta

${ }^{1} \mathrm{La}$ idea sustancial en este enfoque subyace en el hecho de que la sensorización y la actuación de un sistema robótico están estrechamente vinculadas por medio de comportamientos. Estos comportamientos son controladores realimentados realizados sobre cualquier base de procesado (redes neuronales, sistemas expertos, programación tradicional, etc) y que en un ambiente y robot determinado producen una actuación de éste etiquetada como tal comportamiento (seguir otro robot, seguir una luz o evitar obstáculos). de planificación en el enfoque reactivo, la comunidad científica perteneciente a este campo comenzó a dedicar mayores esfuerzos para el desarrollo de arquitecturas cognitivas con mayor grado de interacción entre los módulos, es decir que los diferentes módulos se configuran en forma simultánea y coordinada. En tal sentido, a mediados de los años ochenta, comienzan a cobrar relevancia diferentes investigaciones en el campo, tales como las arquitecturas subsumidas [4] y los agentes de competencia [5]. Así, se conforma lo que se denomina "una nueva tendencia en robótica" [6]. Esta tendencia se posiciona entre la planificación de alto nivel de la IA (Inteligencia Artificial) deliberativa y el control de bajo nivel, a la vez que toma inspiración en la naturaleza y pone especial acento en comportamiento $\mathrm{y}$ reacción rápida $\mathrm{y}$ no en conocimiento $\mathrm{y}$ planificación [3].

Para sintezar, las características más relevantes que presentan las arquitecturas de control que se enmarcan dentro de este enfoque son las siguientes [7]:

* Dado este enfoque, las funciones de sensorización y de actuación de un sistema robot están vinculadas directamente mediante comportamientos.

* Su desarrollo es más rápido que las arquitecturas pertenecientes al enfoque basado en conocimiento, ya que requiere menos procesado de la información.

* Tienen capacidad para tratar situaciones no previstas, ya que depositan mayor confianza en el mundo como fuente de información $\mathrm{y}$ de determinación de sus acciones.

* Aún con la simplicidad del enfoque reactivo, estas arquitecturas escalan en complejidad buscando incorporar otras habilidades en los robots, tales como memoria, aprendizaje $y$ comunicación.

* Dado que no intentan mantener representaciones objetivas del ambiente tienen menos dificultades de representación de los estímulos sensoriales.

La figura 3 ilustra las arquitecturas de control que siguen el enfoque basado en comportamientos con sus correspondientes tareas [8], [9].

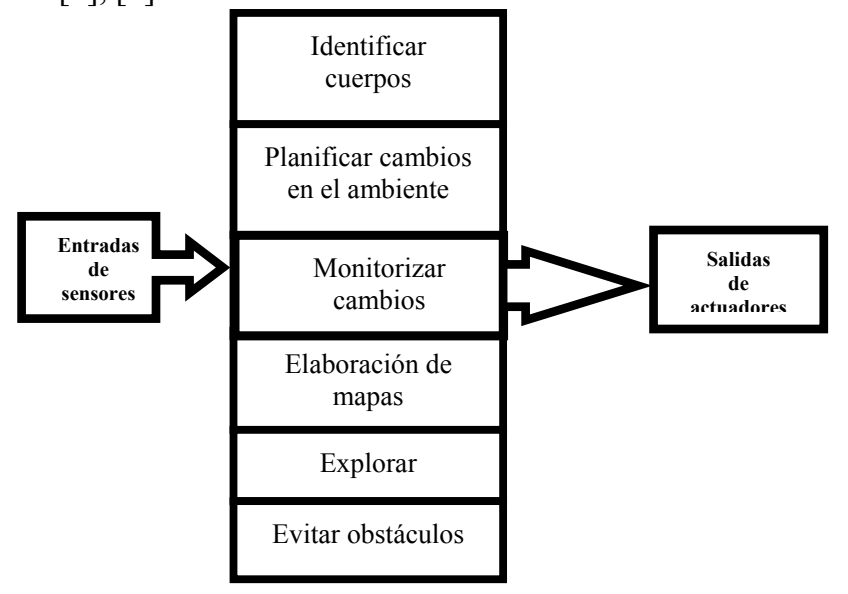

Fig. 3. Arquitectura de control que sigue el enfoque basado en comportamientos 


\section{Enfoque híbrido}

El enfoque se origina en base a la hibridación de los enfoques basados en conocimiento y reactivo, dado a las dificultades que ambos enfoques presentaban por separado. Así, la idea central de este enfoque es obtener las características más significativas que ofrecen los enfoques basados en conocimiento y reactivo por separado, a fin de combinarlas y potenciarlas; dichas características se sintetizan en la figura 4.

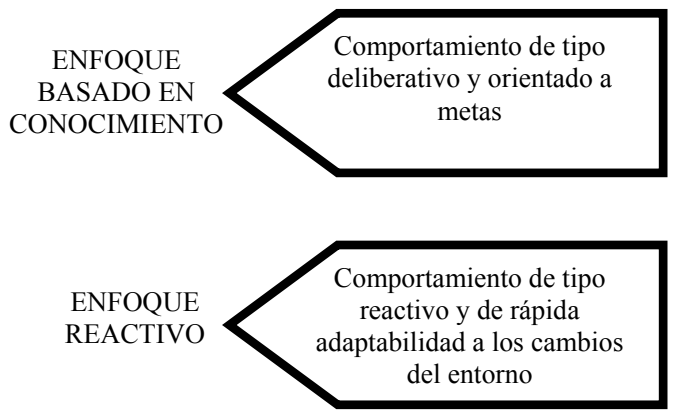

Fig. 4. Características más significativas de los enfoques basados en conocimiento y reactivo

Este enfoque reúne las arquitecturas que brindan cierto compromiso entre las orientadas a metas y las reactivas puras. Las mismas se componen de una "capa de comportamiento" cuya misión consiste en el control de bajo nivel o acciones de base tales como avanzar, retroceder, evitar obstáculos, etc; y una "capa de planificación", encargada de la toma de decisiones de nivel superior o de acciones que requieren mayor elaboración tales como reconocer un objeto $\mathrm{o}$ encaminarse hacia un determinado objetivo. Ambas capas se comunican a los efectos de tener la información correspondiente acerca de las acciones a llevar a cabo y de su ejecución [10].

La figura 3 ilustra las características generales de las arquitecturas de control que siguen el enfoque híbrido.

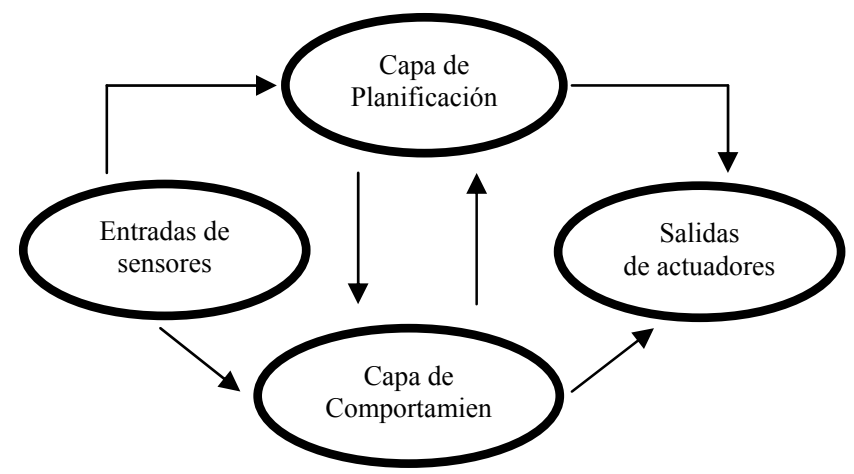

Fig. 5. Arquitectura de control que sigue el enfoque híbrido

Mediante este tipo de arquitecturas se busca optimizar el desempeño del sistema robot en su entorno de navegación en términos de que el mismo sea capaz de esquivar obstáculos, de desenvolverse con una aceptable velocidad de respuesta, se desplace intentando siempre buscar trayectorias óptimas y que alcance los objetivos que le fueron asignados [8].

De este modo, las arquitecturas híbridas son capaces de liberar todo el potencial de las arquitecturas basadas en comportamiento a partir de la incorporación de los mecanismos deliberativos que se llevan a cabo en la capa de planificación y de los mecanismos reactivos que se realizan en la capa de comportamiento; permitiendo de esta forma la reconfiguración de los sistemas de control reactivos por medio de su habilidad para razonar sobre los comportamientos básicos [11].

\section{EXPERIMENTACIÓN CON REDES NEURONALES ARTIFICIALES}

A continuación desarrollaremos un ejemplo práctico de tales conceptos, mediante la simulación de un robot en un entorno determinado, advirtiendo de manera concreta el mecanismo de entrenamiento y los resultados del mismo, en el camino de búsqueda de una trayectoria adecuada y proyectada al desplazamiento del robot desde un origen y hasta un objetivo predeterminado, evitando obstáculos. Dicho en otras palabras, observaremos mediante este ejemplo el proceso que realiza el robot mediante un entorno de simulación con el que éste puede interactuar.

\section{A. Procedimiento para la experimentación}

Como se observa en la figura 6 , el mundo en el que experimenta este vehículo, consta de una grilla de cien espacios, precisamente identificados mediante un eje cartesiano de posición $(\mathrm{x}, \mathrm{y})$, en el que se distribuyen los obstáculos mencionados, presentados con cruces e indicando que por allí el robot no puede avanzar. La línea remarcada en la posición $(9,0)$ describe la llegada u objetivo. A la vez que, en el recuadro de Localización, los indicadores Px y Py, señalan la posición que éste adquiere en su recorrido paso a paso y la orientación con la que se desplaza, a saber: norte, sur, este, oeste. Puede observarse además, que en el diseño del robot se han ilustrado tanto sus sensores y sus motores, en los cuales se actualiza el valor que adquieren durante el proceso de desplazamiento. El objetivo del presente esquema es lograr un seguimiento pormenorizado de la experimentación.

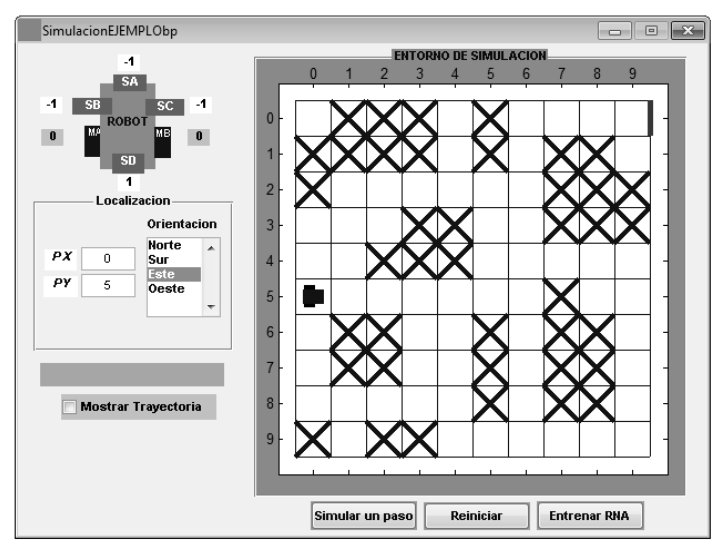

Fig. 6. Entorno de simulación

En consecuencia, se consideran dos tipos de espacios en la grilla: los obstáculos, que no pueden ser ocupados por el robot $\mathrm{y}$ que deben ser evitados por el mismo para no colisionar; $\mathrm{y}$ los libres, aptos para su desplazamiento. Todo el recuadro que delimita la grilla, también es considerado un obstáculo para el robot.

El robot empleado en este ejemplo consta de cuatro sensores, uno en cada cara, dos sensores internos de posición y posee, además, dos motores, cada uno de los cuales ordena el movimiento de las ruedas laterales. 


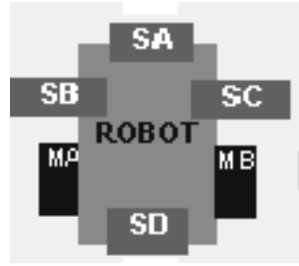

Fig. 7. Esquema Sensor/Motor del Robot

Las coordenadas están dadas por los valores de Px y Py, quienes contienen las coordenadas horizontal $y$ vertical respectivamente, correspondientes a su posición actual en el entorno. Los sensores S1, S2, S3 y S4, son los que deben detectar la proximidad de objetos u obstáculos, adoptando el valor 1 ante la presencia cercana de un objeto (entiéndase por cercano un objeto cuando está a dos casilleros o dos posiciones o menos en relación a la ubicación del sensor, en el entorno que transita el robot); o el valor -1 ante la ausencia cercana de objetos (considerando que un objeto no es cercano cuando está a más de dos casilleros o posiciones respecto del sensor, en el entorno que transita el robot.

De la misma forma, son los motores MA y MB quienes adquieren un par ordenado de valores que producen un efecto determinado, si adoptan el par ordenado $(-1,-1)$, el robot se desplazará una posición hacia atrás, si el par ordenado es $(-1$, 1) el robot se desplazará una posición hacia la izquierda, si es $(1,-1)$, el desplazamiento será una posición a la derecha y si es $(1,1)$ el desplazamiento del robot será una posición hacia delante.

Ya hemos presentado el ambiente para el ejemplo de navegación robótica con redes neuronales artificiales. A continuación, observemos el recorrido esperado desde el origen hasta el destino.

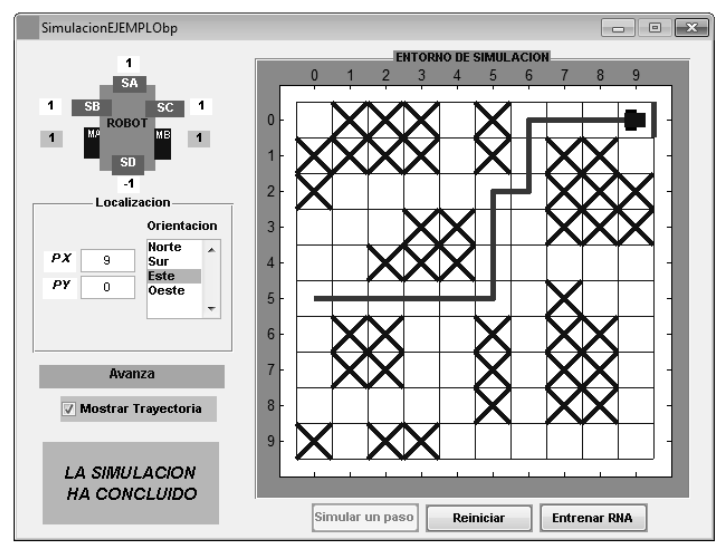

Fig. 8. Trayectoria de entrenamiento del Robot

La figura 6, muestra la posición de partida del robot, que para nuestro caso de estudio es $(0,5)$, y la orientación del mismo, en este caso: Este; indica también, los valores obtenidos para cada sensor y motor, luego de que el cartel enseñe el título "RNA Entrenada!!!"; mientras que la figura 8 muestra, mediante una traza remarcada, la trayectoria del robot que se utilizará para cumplir su objetivo, a través de la búsqueda de patrones de entrenamiento, donde la red neuronal actuará como "cerebro" del robot. El mismo debe llegar a destino, indicado en la posición $(9,0)$.

Para la construcción de un mapa "sensor-motor" para el robot con la trayectoria indicada, se arma una matriz PS (que contiene los datos de los sensores y la posición), y una matriz
$\mathrm{T}$ (que contiene la salida asignada al estado sensorial almacenado en la respectiva fila de $\mathrm{P}$ ).

Haciendo uso de los recursos del entorno propuesto, se entrena una red de tipo Perceptron que consta de seis entradas $\mathrm{y}$ dos neuronas de salida.

Con todos estos datos necesarios para la experimentación, mediante los recursos disponibles de los lenguajes de programación, puede crearse un programa que permite tal simulación, en este caso, nuestro programa ha de llamarse "SimulacionEJEMPLObp".

Para la trayectoria propuesta la figura 8 muestra que el robot puede resolver correctamente el problema de "llegar a destino".

Consecuentemente, para experimentar este aprendizaje del robot, se analiza la salida de la red en otras posiciones.

A continuación, se simula el comportamiento del robot, iniciando su trayectoria en distintas posiciones. Puede observarse que cuando el robot parte de $(0,6)$ pero con orientación Norte en la figura 9, recorre la grilla hasta llegar el final de ella y regresa por el mismo camino hasta encontrar la trayectoria aprendida para llegar al objetivo.

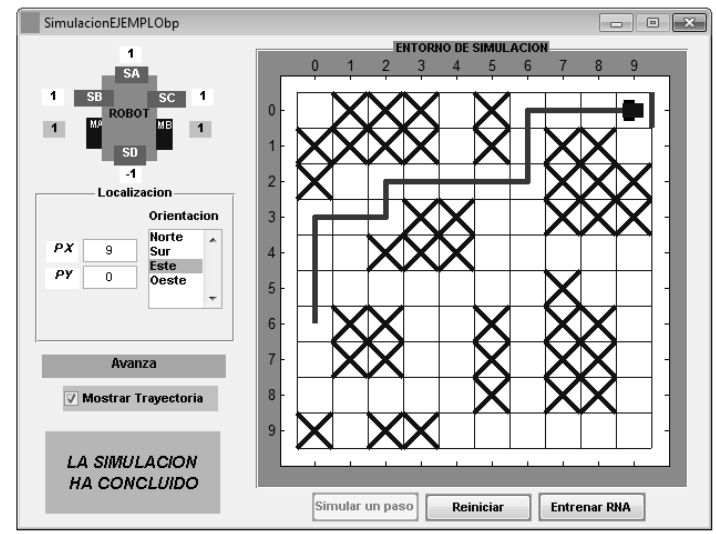

Fig. 9. Trayectoria de Entrenamiento resuelta correctamente por la RNA para un punto de partida $(0,6)$, con orientación Norte

Si ahora orientamos el robot al Este o al Oeste, los resultados obtenidos son los observados en las figuras 10 y 11 ; concluyendo así que no es capaz de alcanzar la trayectoria aprendida y colisiona en ambos casos.

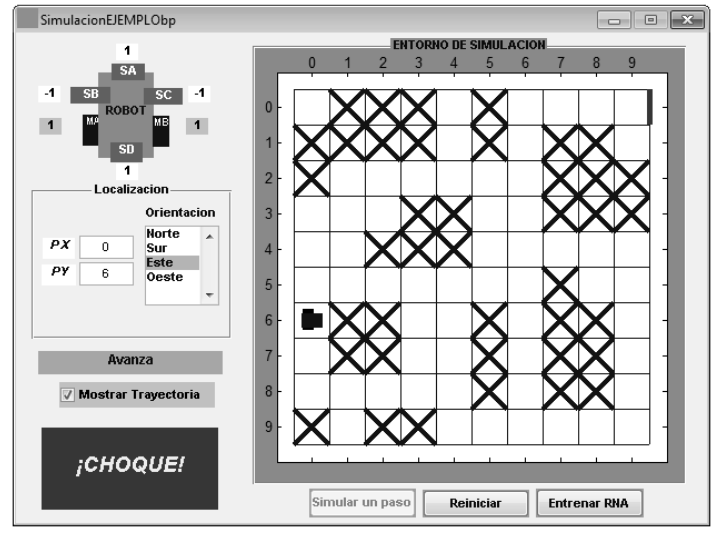

Fig. 10. Trayectoria de Entrenamiento no resuelta por la RNA para un punto de partida $(0,6)$, con orientación Este 


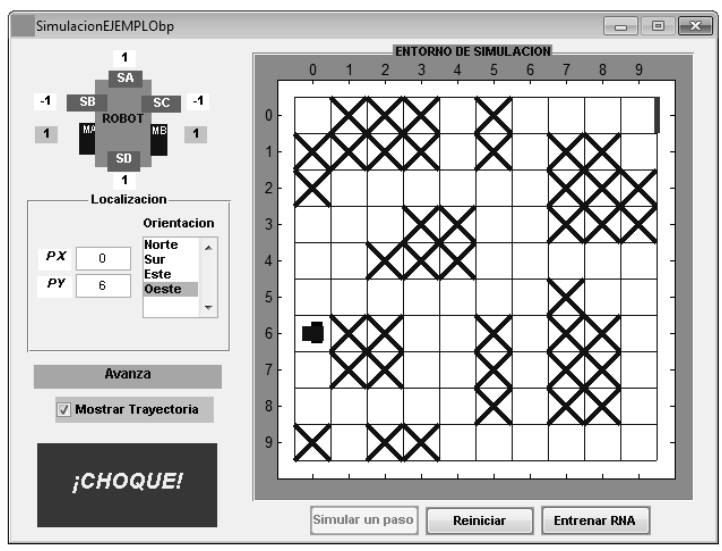

Fig. 11. Trayectoria de Entrenamiento no resuelta por la RNA para un punto de partida $(0,6)$, con orientación Oeste

\section{B. Análisis de los resultados obtenidos}

Una de las primeras inferencias significativas que se puede hacer en función de los resultados que arrojaron los experimentos realizados, es que el patrón de entrenamiento ha sido suficiente para algunas trayectorias que entrenó el robot, pero en líneas generales, este dicho patrón no resulta ser muy representativo del entorno bidimensional en el cual este se desplaza, habida cuenta de que la mayoría de las salidas que manifiestan sus motores consiste en avanzar y por esa razón es que colisiona partiendo desde otras posiciones y/o otra orientación, tal como se muestra en las figuras 10 y 11. Para atenuar este problema, se considera aumentar el número de patrones de entrenamiento utilizando trayectorias más largas, vigilando siempre la convergencia de la red. Conforme a lo expresado, es de hacer notar que, por ejemplo, cuando se considera una trayectoria de entrenamiento como la de la figura 11, la cual está dotada de mayor complejidad en lo que hace a la mayor cantidad de maniobras que debe realizar el robot en su conducta de navegación, se observa que el mismo no puede aprenderla ante la no convergencia de la red propuesta.

Por otra parte, otro detalle importante a considerar en el desarrollo bajo este paradigma, es la constante búsqueda del robot por hallar su trayectoria de entrenamiento; lo cual constituye una conclusión lógica es en conformidad con las características del paradigma reactivo que representan las redes neuronales, pero cabe señalar que en situaciones reales, esta situación puede conllevar a un desgaste excesivo del sistema robot en su accionar navegador, así como también a un alto consumo de combustible.

En síntesis, se asume que el uso de las Redes Neuronales Artificiales como técnica de navegación de características reactivas proporciona resultados satisfactorios para ciertas trayectorias en la fase de operación, tanto más en la medida que estas trayectorias presenten mayor similitud con las que desarrolló en la fase de entrenamiento; así es que se tendrá por caso, que el robot buscará girar más para el lado que lo hace en la trayectoria de entrenamiento que para el otro. A medida que las trayectorias que se le proponen al robot son tanto más complejas que la que este entrenó, este paradigma exhibe sus limitaciones haciendo que la red no converja y se produzcan situaciones de colisión en el ambiente de navegación. En otros términos, se produce una incorrecta generalización de la red neuronal para las nuevas situaciones que debe afrontar el robot, las cuales no se encontraban presentes en las trayectorias de entrenamiento.

\section{CONCLUSIONES}

Las arquitecturas híbridas combinan los métodos de procesamiento reactivo y de planificación a los efectos de poder consolidar un diseño de arquitectura más robusta, tendiente a optimizar el desempeño del robot en el ámbito de su entorno de operación.

La idea que subyace detrás de este objetivo central, es que dichas mejoras puedan verse reflejadas en términos de evitación de obstáculos, velocidad de respuesta, optimización de las trayectorias y logro de los objetivos.

Por otra parte, el comportamiento global del agente robótico está definido por la continua interacción entre las capas que componen la arquitectura.

En síntesis, las arquitecturas híbridas, a través de la combinación de ambos enfoques (basado en conocimiento y basado en comportamiento), permiten la reconfiguración de los sistemas de control reactivos por medio de su habilidad para razonar sobre los comportamientos fundamentales.

Además, y en virtud de todo lo expuesto cabe considerar las siguientes cuestiones sobre las que este grupo de investigación se encuentra trabajando con el objetivo de mejorar el desempeño del robot en lo que se refiere a sus conductas de navegación.

Trabajar con modelos de redes neuronales de arquitecturas más complejas (agregando capas ocultas) para lograr una máxima generalización de la red y de esta manera, poder implementar trayectorias más representativas del ambiente de navegación del robot, teniendo en cuenta que a medida que aumenta la cantidad y complejidad de la información que usa la red es más difícil que esta converja.

Complementando el concepto expresado en el punto anterior, considerar la aplicación de técnicas de razonamiento de alto nivel de tipo deliberativas (aprendizaje automático y planificación autónoma de tareas entre otras) como complemento de las técnicas reactivas; las cuales, si bien son de menor velocidad de reacción que estas, también le permiten al robot afrontar requerimientos de desempeño más complejos.

\section{REFERENCIAS}

[1] Santos, J., Duro, R.: Evolución Artificial y Robótica Autónoma, Ed. Alfaomega - Ra-Ma, México (2005)

[2] Harvey, I.: Artificial Evolution and Real Robots, Proceedings of Internacional Symposium on Artificial Life and Robotics (AROB), Masanori Sugisaka (Ed), Beppu, Japan, pp. 138-141, (1996)

[3] Amalia F. Foka and Panos E. Trahanias. Predictive autonomous robotnavigation. In Proc. of the IEEE/RSJ International Conference on Intelligent Robots and Systems (IROS), pp. 490495 (2002)

[4] Brooks, R.: Achieving Artificial Intelligence through Building Robots, A.I. Memo 898, MIT, AI Lab (1986)

[5] Maes, P.: A Bottom-up Mechanism for Behavior Selection in an Artificial Creature, Proceedings of the First International Conference on Simulation of Adaptive Behavior (SAB90), The Mit Press, pp. 238-246, (1991)

[6] Sharkey, N.: The New Wave in Robot Learning, Robotics and Autonomous System, Vol. 22, pp. 179-185, (1991)

[7] Jones, J.L. and Flynn, A.M.: Mobile Robots: Inspiration to Implementation, A.K. Peters Ltd., Wellesley, MA, (1993) 
[8] J. H. Connel, ISSS: A Hybrid Achitecture Applied to Robot Navigation". Conf. on Robotics and Automation (ICRA-92), pp. 2719-2724. Proc. of the 1992

[9] Mataric, M.J.: Behavior-Based System: Main Properties and Implications, Proceedings of IEEE Internacional Conference on Robotics and Automation, Workshop on Architectures for Intelligence Control Systems, Nice, France, pp. 46-54, (1992)

[10] Ronald C. Arkin, (Behavior-Based Robotics", The MIT Press, (2000)

[11] Adrian. E. Scillato, Daniel. L. Colón y Juan. E. Balbuena: Técnicas de Navegación Híbrida para Navegación de Robots Móviles. Ed. Rama de Estudiantes del IEEE. Tesis de grado para obtener el grado de Ingeniero Electrónico. Universidad Nacional del Comahue.

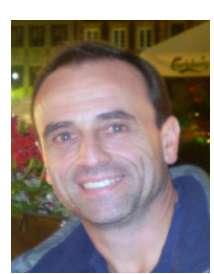

Alejandro Hossian. Ingeniero Civil (Universidad Católica Argentina). Máster en Ingeniería de Software (Universidad Politécnica de Madrid).. Especializado en Sistemas de expertos - Instituto Técnico Buenos Aires (ITBA). Doctor en Ciencias Informáticas, Universidad Nacional de La Plata (2.012). Profesor de la Universidad Tecnológica Nacional - Facultad Regional Neuquén (UTN - FRN). Director del grupo de investigación de robótica y automatización. Sus temas de interés incluyen: robótica, redes neuronales artificiales e Inteligencia Artificial.

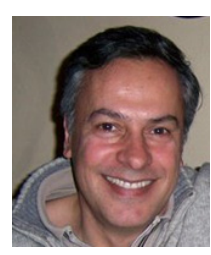

Gustavo Eduardo Monte. Ingeniero Electricista orientación Electrónica Universidad Nacional de Mar del Plata. Master of Science, State University of New York at Stony Brook. Profesor Titular e Investigador de la Universidad Tecnológica Nacional Facultad Regional del Neuquén (UTN - FRN). Sus tópicos de interés incluyen: procesamiento digital de señales, sensores inteligentes y sistemas embebidos. Miembro de la IES Standards Committee de la IEEE.

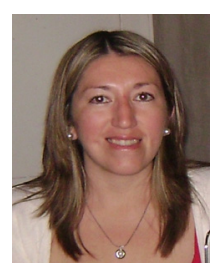

Verónica Olivera. Estudiante avanzado de Ingeniería Electrónica Universidad Tecnológica Nacional - Facultad Regional Neuquén (UTN - FRN). Participó en la elaboración de artículos sobre automatización y Robótica Cognitiva. Ayudante Ad-Honorem de "Tecnología de redes neuronales" en UTN - FRN. Sus temas de interés incluyen: robótica cognitiva, automatización, señales y sistemas, redes neuronales artificiales e Inteligencia Artificial. 